PROCEEDINGS OF THE

AMERICAN MATHEMATICAL SOCIETY

Volume 139, Number 4, April 2011, Pages 1237-1245

S 0002-9939(2010)10550-6

Article electronically published on August 27, 2010

\title{
THE JOHNSON FILTRATION OF THE MCCOOL STABILIZER SUBGROUP OF THE AUTOMORPHISM GROUP OF A FREE GROUP
}

\author{
TAKAO SATOH
}

(Communicated by Richard A. Wentworth)

\begin{abstract}
Let $F_{n}$ be a free group of rank $n$ with basis $x_{1}, x_{2}, \ldots, x_{n}$. We denote by $\mathrm{S}_{n}$ the subgroup of the automorphism group of $F_{n}$ consisting of automorphisms which fix each of $x_{2}, \ldots, x_{n}$ and call it the McCool stabilizer subgroup. Let $\mathrm{IS}_{n}$ be a subgroup of $\mathrm{S}_{n}$ consisting of automorphisms which induce the identity on the abelianization of $F_{n}$. In this paper, we determine the group structure of the lower central series of $\mathrm{IS}_{n}$ and its graded quotients. Then we show that the Johnson filtration of $\mathrm{S}_{n}$ coincides with the lower central series of $\mathrm{IS}_{n}$.
\end{abstract}

\section{INTRODUCTION}

For $n \geq 2$, let $F_{n}$ be a free group of rank $n$ with basis $x_{1}, x_{2}, \ldots, x_{n}$ and $F_{n}=\Gamma_{n}(1), \Gamma_{n}(2), \ldots$ its lower central series. We denote by Aut $F_{n}$ the group of automorphisms of $F_{n}$. For each $k \geq 0$, let $\mathcal{A}_{n}(k)$ be the group of automorphisms of $F_{n}$ which induce the identity on the nilpotent quotient group $F_{n} / \Gamma_{n}(k+1)$. The group $\mathcal{A}_{n}(1)$ is called the IA-automorphism group and is also denoted by $\mathrm{IA}_{n}$. Then we have a descending filtration

$$
\text { Aut } F_{n}=\mathcal{A}_{n}(0) \supset \mathcal{A}_{n}(1) \supset \mathcal{A}_{n}(2) \supset \cdots
$$

of Aut $F_{n}$, called the Johnson filtration of Aut $F_{n}$. The Johnson filtration of Aut $F_{n}$ was originally introduced in 1963 through the remarkable pioneer work by Andreadakis [1, who showed that $\mathcal{A}_{n}(1), \mathcal{A}_{n}(2), \ldots$ is a descending central series of $\mathcal{A}_{n}(1)$ and that for each $k \geq 1$ the graded quotient $\operatorname{gr}^{k}\left(\mathcal{A}_{n}\right):=\mathcal{A}_{n}(k) / \mathcal{A}_{n}(k+1)$ is a free abelian group of finite rank. In general, determining the structure of $\operatorname{gr}^{k}\left(\mathcal{A}_{n}\right)$ plays an important role on the study of the algebraic structure of Aut $F_{n}$. For $1 \leq k \leq 3$, the rank of $\operatorname{gr}^{k}\left(\mathcal{A}_{n}\right)$ has been determined. Andreadakis 1 computed the rank of $\operatorname{gr}^{1}\left(\mathcal{A}_{n}\right)$. Moreover, by independent works of Cohen-Pakianathan [4, 5], Farb [6] and Kawazumi [10, it is known that $\operatorname{gr}^{1}\left(\mathcal{A}_{n}\right)$ is isomorphic to the abelianization of $\mathrm{IA}_{n}$. For $k=2$ and 3 , the rank of $\operatorname{gr}^{k}\left(\mathcal{A}_{n}\right)$ is determined by Pettet [19]

Received by the editors September 6, 2009 and, in revised form, February 19, 2010 and April 20, 2010.

2010 Mathematics Subject Classification. Primary 20F28; Secondary 20 F14.

Key words and phrases. Automorphism group of a free group, IA-automorphism group, Johnson filtration.

(C)2010 American Mathematical Society Reverts to public domain 28 years from publication 
and Satoh [21] respectively. For $k \geq 4$, however, it seems that there are few results for the structure of $\operatorname{gr}^{k}\left(\mathcal{A}_{n}\right)$.

In the study of the Johnson filtration of Aut $F_{n}$, it would also be interesting to determine whether $\mathcal{A}_{n}(1), \mathcal{A}_{n}(2), \ldots$ coincides with the lower central series $\mathcal{A}_{n}^{\prime}(1), \mathcal{A}_{n}^{\prime}(2), \ldots$ of $\mathcal{A}_{n}(1)$ or not. Andreadakis [1] showed that $\mathcal{A}_{2}(k)=\mathcal{A}_{2}^{\prime}(k)$ and $\mathcal{A}_{3}(3)=\mathcal{A}_{3}^{\prime}(3)$. From the results due to Cohen-Pakianathan [4, 5], Farb [6] and Kawazumi [10, we have $\mathcal{A}_{n}(2)=\mathcal{A}_{n}^{\prime}(2)$ for $n \geq 3$. Furthermore, Pettet [19] obtained that $\mathcal{A}_{n}^{\prime}(3)$ has finite index in $\mathcal{A}_{n}(3)$. Now it is conjectured by Andreadakis that $\mathcal{A}_{n}(k)=\mathcal{A}_{n}^{\prime}(k)$ for any $n \geq 3$ and $k \geq 3$.

In this paper, we give an affirmative answer to the problem above for a certain subgroup of Aut $F_{n}$. Let $\mathrm{S}_{n}$ be the subgroup of Aut $F_{n}$ consisting of automorphisms which fix each of $x_{2}, \ldots, x_{n}$. We call $\mathrm{S}_{n}$ the McCool stabilizer subgroup of Aut $F_{n}$. Let $\mathrm{IS}_{n}$ be the subgroup of $\mathrm{S}_{n}$ consisting of automorphisms which induce the identity on the abelianization of $F_{n}$. The groups $\mathrm{S}_{n}$ and $\mathrm{IS}_{n}$ were first studied by McCool. He 14] gave a finite presentation of $S_{n}$ and showed that $\mathrm{IS}_{n}$ is not finitely presentable. Furthermore, he [14] also gave an infinite presentation of $\mathrm{IS}_{n}$. Set $\mathcal{S}_{n}(k):=\mathcal{A}_{n}(k) \cap \mathrm{S}_{n}$ for each $k \geq 0$. Then $\mathcal{S}_{n}(0)=\mathrm{S}_{n}$ and $\mathcal{S}_{n}(1)=\mathrm{IS}_{n}$. We call a descending central filtration

$$
\mathrm{S}_{n}=\mathcal{S}_{n}(0) \supset \mathcal{S}_{n}(1) \supset \mathcal{S}_{n}(2) \supset \cdots
$$

the Johnson filtration of $\mathrm{S}_{n}$. On the other hand, we also consider the lower central series of $\mathrm{IS}_{n}$, denoted by $\mathcal{S}_{n}^{\prime}(1), \mathcal{S}_{n}^{\prime}(2), \ldots$. Since the Johnson filtration is central, we see $\mathcal{S}_{n}^{\prime}(k) \subset \mathcal{S}_{n}(k)$ in general. The main theorem of the paper is

Theorem 1 (= Theorem 4.1). For each $k \geq 1$, we have $\mathcal{S}_{n}(k)=\mathcal{S}_{n}^{\prime}(k)$.

In order to show this, we study the group structure of the lower central series $\mathcal{S}_{n}^{\prime}(k)$. Let $F$ be a subgroup of $F_{n}$ generated by $x_{2}, \ldots, x_{n}$. The group $F$ is a free group of rank $n-1$. Let $\Gamma_{F}(1), \Gamma_{F}(2), \ldots$ be the lower central series of $F$. In Section 3, we show that $\mathcal{S}_{n}^{\prime}(k)$ is isomorphic to the semidirect product

$$
\mathcal{S}_{n}^{\prime}(k) \cong \Gamma_{F}(k+1) \rtimes \Gamma_{F}(k)
$$

of $\Gamma_{F}(k+1)$ and $\Gamma_{F}(k)$ for each $k \geq 1$. (See Lemma 3.1.) We remark that McCool

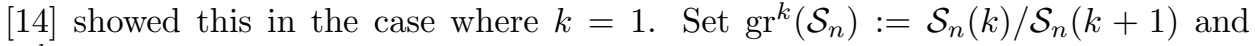
$\operatorname{gr}^{k}\left(\mathcal{S}_{n}^{\prime}\right):=\mathcal{S}_{n}^{\prime}(k) / \mathcal{S}_{n}^{\prime}(k+1)$ for each $k \geq 1$. Then, using the fact above, we obtain

Corollary 1 (= Corollary 4.1). For each $k \geq 1$,

$$
\operatorname{rank}_{\mathbf{Z}}\left(\operatorname{gr}^{k}\left(\mathcal{S}_{n}\right)\right)=\operatorname{rank}_{\mathbf{Z}}\left(\operatorname{gr}^{k}\left(\mathcal{S}_{n}^{\prime}\right)\right)=r_{n-1}(k+1)+r_{n-1}(k),
$$

where

$$
r_{n}(k):=\operatorname{rank}_{\mathbf{Z}}\left(\Gamma_{n}(k) / \Gamma_{n}(k+1)\right) .
$$

Here we remark that each of $r_{n}(k)$ has been determined by Witt 22. (See Subsection 2.3 for details.)

In general, it is also not determined whether each subgroup $\mathcal{A}_{n}(k)$ of the Johnson filtration of Aut $F_{n}$ is finitely genrated or not. It is still a difficult open problem. It is, however, easily seen that for any $n \geq 3$ and $k \geq 2$, the group $\mathcal{S}_{n}(k)$ is not finitely generated. More precisely, from (1) and Corollary 11 we have

$$
H_{1}\left(\mathcal{S}_{n}(k), \mathbf{Z}\right) \cong\left(\Gamma_{F}(k+1) /\left[\Gamma_{F}(k+1), \Gamma_{F}(k)\right]\right) \oplus H_{1}\left(\Gamma_{F}(k), \mathbf{Z}\right) .
$$

(See Corollary 3.2.) 
This paper consists of five sections. In Section 2, we recall the IA-automorphism group and the Johnson filtration of the automorphism group of a free group. In Section 3, we study the McCool stabilizer subgroup. In Section 4, we show that the Johnson filtration of $\mathrm{S}_{n}$ coincides with its lower central series of $\mathrm{IS}_{n}$.

\section{Preliminaries}

In this section, we recall the definition and some properties of the IAautomorphism group and of the Johnson homomorphisms of the automorphism group of a free group.

2.1. Notation. Throughout the paper, we use the following notation and conventions. Let $G$ be a group and $N$ a normal subgroup of $G$.

- The abelianization of $G$ is denoted by $G^{\text {ab }}$.

- The group automorphism group Aut $G$ of $G$ acts on $G$ from the right. For any $\sigma \in$ Aut $G$ and $x \in G$, the action of $\sigma$ on $x$ is denoted by $x^{\sigma}$.

- For an element $g \in G$, we also denote the coset class of $g$ by $g \in G / N$ without any confusion.

- For elements $x$ and $y$ of $G$, the commutator bracket $[x, y]$ of $x$ and $y$ is defined to be $[x, y]:=x y x^{-1} y^{-1}$.

2.2. IA-automorphism group. For $n \geq 2$, let $F_{n}$ be a free group of rank $n$ with basis $x_{1}, \ldots, x_{n}$. We denote the abelianization of $F_{n}$ by $H$ and its dual group by $H^{*}:=\operatorname{Hom}_{\mathbf{Z}}(H, \mathbf{Z})$. Let $\rho: \operatorname{Aut} F_{n} \rightarrow$ Aut $H$ be the natural homomorphism induced from the abelianization of $F_{n}$. In this paper we identify Aut $H$ with the general linear group $\mathrm{GL}(n, \mathbf{Z})$ by fixing the basis of $H$ as a free abelian group induced from the basis $x_{1}, \ldots, x_{n}$ of $F_{n}$. The kernel $\mathrm{IA}_{n}$ of $\rho$ is called the IAautomorphism group of $F_{n}$. It is known due to Nielsen 18 that $\mathrm{IA}_{2}$ coincides with the inner automorphism group Inn $F_{2}$ of $F_{2}$. Namely, IA 2 is a free group of rank 2. However, $\mathrm{IA}_{n}$ for $n \geq 3$ is much larger than the inner automorphism group $\operatorname{Inn} F_{n}$. Indeed, Magnus [12] showed that for any $n \geq 3, \mathrm{IA}_{n}$ is finitely generated by automorphisms

$$
K_{i j}:\left\{\begin{aligned}
x_{i} & \mapsto x_{j}{ }^{-1} x_{i} x_{j}, \\
x_{t} & \mapsto x_{t},
\end{aligned} \quad(t \neq i)\right.
$$

for distinct $i, j \in\{1,2, \ldots, n\}$ and

$$
K_{i j k}:\left\{\begin{array}{lll}
x_{i} & \mapsto x_{i} x_{j} x_{k} x_{j}{ }^{-1} x_{k}{ }^{-1}, \\
x_{t} & \mapsto x_{t}, \quad(t \neq i)
\end{array}\right.
$$

for distinct $i, j, k \in\{1,2, \ldots, n\}$ such that $j>k$.

For any $n \geq 3$, although a generating set of $\mathrm{IA}_{n}$ is obtained as mentioned above, any presentation of $\mathrm{IA}_{n}$ is still not known. For $n=3$, Krstić and McCool [1] showed that $\mathrm{IA}_{3}$ is not finitely presentable. For $n \geq 4$, it is not known whether $\mathrm{IA}_{n}$ is finitely presentable or not. Recently, Cohen-Pakianathan [4, 5], Farb [6] and Kawazumi [10] independently showed

$$
\mathrm{IA}_{n}^{\mathrm{ab}} \cong H^{*} \otimes \mathbf{z} \Lambda^{2} H
$$

as a $\operatorname{GL}(n, \mathbf{Z})$-module. 
2.3. Free Lie algebras. In this subsection we recall the free Lie algebra. Let $\Gamma_{n}(1) \supset \Gamma_{n}(2) \supset \cdots$ be the lower central series of a free group $F_{n}$ defined by the rule

$$
\Gamma_{n}(1):=F_{n}, \quad \Gamma_{n}(k):=\left[\Gamma_{n}(k-1), F_{n}\right], \quad k \geq 2 .
$$

We denote by $\mathcal{L}_{n}(k):=\Gamma_{n}(k) / \Gamma_{n}(k+1)$ the graded quotient of the lower central series of $F_{n}$ and by $\mathcal{L}_{n}:=\bigoplus_{k>1} \mathcal{L}_{n}(k)$ the associated graded sum. Since the group Aut $F_{n}$ naturally acts on $\mathcal{L}_{n}(k)$ for each $k \geq 1$ and since $\mathrm{IA}_{n}$ acts on it trivially, the action of $\operatorname{GL}(n, \mathbf{Z})$ on each $\mathcal{L}_{n}(k)$ is well-defined. Furthermore, the graded sum $\mathcal{L}_{n}$ naturally has a graded Lie algebra structure induced from the commutator bracket on $F_{n}$ and is called the free Lie algebra generated by $H$. (See 20] for basic material concerning free Lie algebras.) It is classically well known due to Witt 22] that each $\mathcal{L}_{n}(k)$ is a $\mathrm{GL}(n, \mathbf{Z})$-equivariant free abelian group of rank

$$
r_{n}(k):=\frac{1}{k} \sum_{d \mid k} \mu(d) n^{\frac{k}{d}},
$$

where $\mu$ is the Möbius function. For example, the $\operatorname{GL}(n, \mathbf{Z})$-module structure of $\mathcal{L}_{n}(k)$ for $1 \leq k \leq 3$ is given by

$$
\begin{aligned}
& \mathcal{L}_{n}(1)=H, \quad \mathcal{L}_{n}(2)=\Lambda^{2} H, \\
& \mathcal{L}_{n}(3)=\left(H \otimes_{\mathbf{z}} \Lambda^{2} H\right) /\langle x \otimes y \wedge z+y \otimes z \wedge x+z \otimes x \wedge y \mid x, y, z \in H\rangle .
\end{aligned}
$$

Next, we consider an embedding of the free Lie algebra into the tensor algebra. Let $T(H)$ be the tensor algebra of $H$ over $\mathbf{Z}$. Then $T(H)$ is the universal enveloping algebra of the free Lie algebra $\mathcal{L}_{n}$, and the natural map $\iota: \mathcal{L}_{n} \rightarrow T(H)$ defined by

$$
[X, Y] \mapsto X \otimes Y-Y \otimes X
$$

for $X, Y \in \mathcal{L}_{n}$ is an injective graded Lie algebra homomorphism. We denote by $\iota_{k}$ the homomorphism of degree $k$ part of $\iota$ and consider $\mathcal{L}_{n}(k)$ as a submodule $H^{\otimes k}$ through $\iota_{k}$.

Now, we consider a Lie subalgebra of $\mathcal{L}_{n}$ generated by $x_{2}, \ldots, x_{n}$. Let $F$ be a subgroup of $F_{n}$ generated by $x_{2}, x_{3}, \ldots, x_{n}$. The group $F$ is a free group of rank $n-1$. We denote the lower central series of $F$ by $\Gamma_{F}(1), \Gamma_{F}(2), \cdots$ and write $\mathcal{L}_{F}(k)$ for its graded quotient $\Gamma_{F}(k) / \Gamma_{F}(k+1)$ for each $k \geq 1$. Clearly, $\mathcal{L}_{F}(k) \cong \mathcal{L}_{n-1}(k)$ as an abelian group. The associated graded sum $\mathcal{L}_{F}:=\bigoplus_{k \geq 1} \mathcal{L}_{F}(k) / \mathcal{L}_{F}(k+1)$ also has a graded Lie algebra structure. By the elimination theorem of free Lie algebras, we see that $\mathcal{L}_{F}$ is a direct summand of $\mathcal{L}_{n}$. (See Proposition 10 in 3.) Hence, in particular, $\mathcal{L}_{F}(k)$ is a direct summand of $\mathcal{L}_{n}(k)$ for each $k \geq 1$.

2.4. Johnson filtration. In this subsection, we recall the Johnson filtration of Aut $F_{n}$. For $k \geq 0$, the action of Aut $F_{n}$ on each nilpotent quotient $F_{n} / \Gamma_{n}(k+1)$ induces a homomorphism

$$
\text { Aut } F_{n} \rightarrow \operatorname{Aut}\left(F_{n} / \Gamma_{n}(k+1)\right) \text {. }
$$

We denote the kernel of the homomorphism above by $\mathcal{A}_{n}(k)$. Then the groups $\mathcal{A}_{n}(k)$ define a descending central filtration

$$
\text { Aut } F_{n}=\mathcal{A}_{n}(0) \supset \mathcal{A}_{n}(1) \supset \mathcal{A}_{n}(2) \supset \cdots
$$

of Aut $F_{n}$, with $\mathcal{A}_{n}(1)=I A_{n}$. We call it the Johnson filtration of Aut $F_{n}$. For each $k \geq 1$, the group Aut $F_{n}$ acts on $\mathcal{A}_{n}(k)$ by conjugation, and it naturally induces an action of $\operatorname{GL}(n, \mathbf{Z})$ on $\operatorname{gr}^{k}\left(\mathcal{A}_{n}\right):=\mathcal{A}_{n}(k) / \mathcal{A}_{n}(k+1)$. The graded sum 
$\operatorname{gr}\left(\mathcal{A}_{n}\right):=\bigoplus_{k \geq 1} \operatorname{gr}^{k}\left(\mathcal{A}_{n}\right)$ has a graded Lie algebra structure induced from the commutator bracket on $\mathrm{IA}_{n}$.

In order to study the graded quotients $\operatorname{gr}^{k}\left(\mathcal{A}_{n}\right)$, the Johnson homomorphisms are used. For each $k \geq 1$, define a homomorphism $\mathcal{A}_{n}(k) \rightarrow \operatorname{Hom}_{\mathbf{Z}}\left(H, \mathcal{L}_{n}(k+1)\right)$ by

$$
\sigma \mapsto\left(x \mapsto x^{-1} x^{\sigma}\right), \quad x \in H .
$$

Then the kernel of this homomorphism is just $\mathcal{A}_{n}(k+1)$. Hence it induces an injective homomorphism

$$
\tau_{k}: \operatorname{gr}^{k}\left(\mathcal{A}_{n}\right) \hookrightarrow \operatorname{Hom}_{\mathbf{Z}}\left(H, \mathcal{L}_{n}(k+1)\right) .
$$

The homomorphism $\tau_{k}$ is called the $k$-th Johnson homomorphism of Aut $F_{n}$.

Historically, the study of the Johnson homomorphisms was begun in 1980 by D. Johnson [8. He studied the Johnson homomorphism of a mapping class group of a closed oriented surface and determined the abelianization of the Torelli group. (See 9.) There is a broad range of remarkable results for the Johnson homomorphisms of a mapping class group. (For example, see [7] and [15, [16, 17.)

In general, since $\tau_{k}$ is injective, to determine the image (or equivalently, the cokernel) of $\tau_{k}$ is an important problem in the study of the structure of $\operatorname{gr}^{k}\left(\mathcal{A}_{n}\right)$. In this paper, we use the Johnson homomorphism only in the proof of Theorem 4.1.

\section{McCool StABILIZER SUbGroup}

Here we consider the McCool stabilizer subgroup. In the following, we assume $n \geq 3$. Let $\mathrm{S}_{n}$ be the subgroup of Aut $F_{n}$ consisting of automorphisms which fix each of $x_{2}, \ldots, x_{n}$. We call $\mathrm{S}_{n}$ the McCool stabilizer subgroup. We denote the intersection of $\mathrm{S}_{n}$ with $\mathrm{IA}_{n}$ by $\mathrm{IS}_{n}$. McCool [14 showed that $\mathrm{IS}_{n}$ is finitely generated but not finitely presentable. He [14] also gave an infinite presentation of $\mathrm{IS}_{n}$.

For any $i \in\{2, \ldots, n\}$, let $v_{i}$ be the automorphism of $F_{n}$ which sends $x_{1}$ to $x_{1} x_{i}$ and fix the other generators $x_{t}$. The subgroup $V$ of Aut $F_{n}$ generated by all $v_{i}$ is a free group of rank $n-1$. The subgroup $W$ of $\mathrm{IA}_{n}$ generated by all $K_{1 i}$ is also a free group of rank $n-1$. Then McCool [14] showed that $\mathrm{IS}_{n}$ is a semidirect product of $[V, V]$ by $W$. Namely, we have a split group extension

$$
1 \rightarrow[V, V] \rightarrow \mathrm{IS}_{n} \rightarrow W \rightarrow 1 .
$$

Furthermore, in [14] he showed that $[V, V]$ is the normal closure of $\left\{K_{1 i j} \mid i>j\right\}$ in $\mathrm{IS}_{n}$, and $\mathrm{IS}_{n}$ is generated by $K_{1 i}$ and $K_{1 i j}$. Thus, considering a homomorphism $\mathrm{IS}_{n} \hookrightarrow \mathrm{IA}_{n} \rightarrow \mathrm{IA}^{\mathrm{ab}} \cong H^{*} \otimes_{\mathbf{Z}} \Lambda^{2} H$, we see that $H_{1}\left(\mathrm{IS}_{n}, \mathbf{Z}\right)$ is a free abelian group of rank $n(n-1) / 2$ with basis $\left\{K_{1 i}, K_{1 j k} \mid 2 \leq i, j, k \leq n, j>k\right\}$.

In this paper, we consider the Johnson filtration of Aut $F_{n}$ restricted to $\mathrm{S}_{n}$. Namely, set $\mathcal{S}_{n}(k):=\mathcal{A}_{n}(k) \cap \mathrm{S}_{n}$ for each $k \geq 0$. Then $\mathcal{S}_{n}(0)=\mathrm{S}_{n}$ and $\mathcal{S}_{n}(1)=\mathrm{IS}_{n}$. We call a descending central filtration

$$
\mathrm{S}_{n}=\mathcal{S}_{n}(0) \supset \mathcal{S}_{n}(1) \supset \mathcal{S}_{n}(2) \supset \cdots
$$

the Johnson filtration of $\mathrm{S}_{n}$. Set $\operatorname{gr}^{k}\left(\mathcal{S}_{n}\right):=\mathcal{S}_{n}(k) / \mathcal{S}_{n}(k+1)$. We denote by $\tau_{k}^{S}$ the restriction of the Johnson homomorphism $\tau_{k}$ to $\operatorname{gr}^{k}\left(\mathcal{S}_{n}\right) \subset \operatorname{gr}^{k}\left(\mathcal{A}_{n}\right)$ and call it the Johnson homomorphism of $\mathrm{S}_{n}$.

Let $\mathcal{S}_{n}^{\prime}(1) \supset \mathcal{S}_{n}^{\prime}(2) \supset \cdots$ be the lower central series of $\operatorname{IS}_{n}=\mathcal{S}_{n}(1)$. Clearly, we have $\mathcal{S}_{n}^{\prime}(k) \subset \mathcal{S}_{n}(k)$ for each $k \geq 1$. Here we determine the group structure of $\mathcal{S}_{n}^{\prime}(k)$. To do this, we prepare some notation. For any $x \in F$, let $v_{x}$ be an 
automorphism of $F_{n}$ which maps $x_{1}$ to $x_{1} x$ and fixes the other generators $x_{t}$. Then a map $\psi_{V}: F \rightarrow V$ defined by $\psi_{V}(x):=v_{x}$ is an isomorphism. Similarly, for any $y \in F$, let $w_{y}$ be an automorphism of $F_{n}$ which maps $x_{1}$ to $y^{-1} x_{1} y$ and fixes the other generators $x_{t}$. Then a map $\psi_{W}: F \rightarrow W$ defined by $\psi_{W}(y):=w_{y}$ is also an isomorphism. We denote the lower central series of $V$ and $W$ by $\Gamma_{V}(k)$ and $\Gamma_{W}(k)$ respectively. Then we have

Lemma 3.1. For each $k \geq 1, \mathcal{S}_{n}^{\prime}(k)$ is a semidirect product of $\Gamma_{V}(k+1)$ and $\Gamma_{W}(k)$ :

$$
\mathcal{S}_{n}^{\prime}(k)=\Gamma_{V}(k+1) \rtimes \Gamma_{W}(k) .
$$

Proof. It is easily seen that

- $\Gamma_{W}(k)$ is a subgroup of $\mathcal{S}_{n}^{\prime}(k)$,

- $\Gamma_{V}(k+1)$ is a normal subgroup of $\mathcal{S}_{n}^{\prime}(k)$ and

- $\Gamma_{V}(k+1) \cap \Gamma_{W}(k)=1$

for each $k \geq 1$. We leave proofs of them to the reader as exercises. Hence it suffices to show that $\mathcal{S}_{n}^{\prime}(k)=\Gamma_{V}(k+1) \Gamma_{W}(k)$. We prove this by induction on $k$. It is clear for $k=1$ by (44). Suppose $k \geq 2$. By the inductive hypothesis, we have $\mathcal{S}_{n}^{\prime}(k-1)=\Gamma_{V}(k) \Gamma_{W}(k-1)$. It suffices to show that for any $\sigma \in \mathcal{S}_{n}^{\prime}(k-1)$ and $\sigma^{\prime} \in \mathcal{S}_{n}^{\prime}(1)$, the commutator $\left[\sigma, \sigma^{\prime}\right]$ is in $\Gamma_{V}(k+1) \Gamma_{W}(k)$ since $\mathcal{S}_{n}^{\prime}(k)$ is generated by all elements of type $\left[\sigma, \sigma^{\prime}\right]$.

Let $\sigma=v w$ and $\sigma^{\prime}=v^{\prime} w^{\prime}$ for $v \in \Gamma_{V}(k), v^{\prime} \in \Gamma_{V}(2), w \in \Gamma_{W}(k-1)$ and $w^{\prime} \in \Gamma_{W}(1)$. Then we see that

$$
\begin{aligned}
{\left[\sigma, \sigma^{\prime}\right] } & =\left[v w, v^{\prime} w^{\prime}\right] \\
& =v\left(w v^{\prime} w^{-1}\right)\left(w w^{\prime} w^{-1} v^{-1} w w^{-1} w^{-1}\right)\left(\left[w, w^{\prime}\right] v^{\prime-1}\left[w^{\prime}, w\right]\right)\left[w, w^{\prime}\right] .
\end{aligned}
$$

Since $\left[w, w^{\prime}\right] \in \Gamma_{W}(k)$, we show that

$$
\sigma^{\prime \prime}:=v\left(w v^{\prime} w^{-1}\right)\left(w w^{\prime} w^{-1} v^{-1} w w^{\prime-1} w^{-1}\right)\left(\left[w, w^{\prime}\right] v^{\prime-1}\left[w^{\prime}, w\right]\right) \in \Gamma_{V}(k+1) .
$$

Now, set

$$
v=v_{x}, \quad v^{\prime}=v_{x^{\prime}}, \quad w=w_{y}, \quad w^{\prime}=w_{y^{\prime}}
$$

for $x \in \Gamma_{F}(k), x^{\prime} \in \Gamma_{F}(2), y \in \Gamma_{F}(k-1)$ and $y^{\prime} \in \Gamma_{F}(1)$. Then we have

$$
x_{1}^{\sigma^{\prime \prime}}=x_{1}\left(\left[y, y^{\prime}\right] x^{-1}\left[y^{\prime}, y\right]\right)\left(y y^{\prime} y^{-1} x^{-1} y y^{\prime-1} y^{-1}\right)\left(y x^{\prime} y^{-1}\right) x .
$$

Therefore it suffices to show that

$$
z:=\left(\left[y, y^{\prime}\right] x^{-1}\left[y^{\prime}, y\right]\right)\left(y y^{\prime} y^{-1} x^{-1} y y^{\prime-1} y^{-1}\right)\left(y x^{\prime} y^{-1}\right) x \in \Gamma_{F}(k+1) .
$$

Since $x^{ \pm 1},\left[y, y^{\prime}\right] \in \Gamma_{F}(k)$ commutes with $x^{\prime}, y$ and $y^{\prime}$ modulo $\Gamma_{F}(k+1)$,

$$
z \equiv\left(\left[y, y^{\prime}\right] x^{\prime^{-1}}\left[y^{\prime}, y\right]\right)\left(y x^{\prime} y^{-1}\right) \equiv x^{\prime-1}\left(y x^{\prime} y^{-1}\right)=\left[x^{\prime-1}, y\right] \equiv 0
$$

modulo $\Gamma_{F}(k+1)$. Hence we obtain

$$
\sigma^{\prime \prime}=\psi_{V}(z) \in \Gamma_{V}(k+1) .
$$

This completes the proof of Lemma 3.1

From Lemma 3.1, we see that $\sigma \in \mathcal{S}_{n}^{\prime}(k)$ if and only if

$$
x_{1}^{\sigma}=y^{-1} x_{1} y x
$$


for some $x \in \Gamma_{F}(k+1)$ and $y \in \Gamma_{F}(k)$. Furthermore, for any $v_{x} \in \Gamma_{V}(k+1)$ and $w_{y} \in \Gamma_{W}(k)$ for $x \in \Gamma_{F}(k+1)$ and $y \in \Gamma_{F}(k)$, we have

(5) $w_{y}^{-1} v_{x} w_{y}=v_{y^{-1} x y} \in \Gamma_{V}(k+1)$ and $\left[w_{y}, v_{x}\right]=v_{\left[x^{-1}, y^{-1}\right]} \in\left[\Gamma_{V}(k+1), \Gamma_{V}(k)\right]$.

In particular,

$$
w_{y}^{-1} v_{x} w_{y} \equiv v_{x} \quad\left(\bmod \Gamma_{V}(k+2)\right) .
$$

Using Lemma 3.1, we can determine the group structure of the graded quotients of the lower central series $\mathcal{S}_{n}^{\prime}(k)$. Set $\operatorname{gr}^{k}\left(\mathcal{S}_{n}^{\prime}\right):=\mathcal{S}_{n}^{\prime}(k) / \mathcal{S}_{n}^{\prime}(k+1)$ for each $k$.

Proposition 3.1. For each $k \geq 1, \operatorname{gr}^{k}\left(\mathcal{S}_{n}^{\prime}\right) \cong \mathcal{L}_{F}(k+1) \oplus \mathcal{L}_{F}(k)$ as a $\mathbf{Z}$-module.

Proof. Consider a surjective map $\Gamma_{V}(k+1) \rtimes \Gamma_{W}(k) \rightarrow \mathcal{L}_{F}(k+1) \oplus \mathcal{L}_{F}(k)$ defined by

$$
v w \mapsto\left(\psi_{V}^{-1}(v), \psi_{W}^{-1}(w)\right) .
$$

Since $\Gamma_{W}(k)$ acts trivially on $\mathcal{L}_{F}(k+1)$ from ([6) , this map is a homomorphism whose kernel is exactly $\Gamma_{V}(k+2) \rtimes \Gamma_{W}(k+1)$. Hence we obtain Proposition 3.1 .

As a corollary, we have

Corollary 3.1. For each $k \geq 1, \operatorname{rank}_{\mathbf{Z}}\left(\operatorname{gr}^{k}\left(\mathcal{S}_{n}^{\prime}\right)\right)=r_{n-1}(k+1)+r_{n-1}(k)$.

Finally, we remark that the abelianization of $\mathcal{S}_{n}(k)^{\prime}$ is not finitely generated. More precisely, we have

Corollary 3.2. For each $n \geq 3$ and $k \geq 2$,

$$
H_{1}\left(\mathcal{S}_{n}^{\prime}(k), \mathbf{Z}\right) \cong\left(\Gamma_{F}(k+1) /\left[\Gamma_{F}(k+1), \Gamma_{F}(k)\right]\right) \oplus H_{1}\left(\Gamma_{F}(k), \mathbf{Z}\right) .
$$

Proof. This equation immediately follows from Lemma 3.1 and (5).

\section{The Johnson FiLtration of $\mathrm{IS}_{n}$}

In this section, we show that the Johnson filtaration $\mathcal{S}_{n}(1) \supset \mathcal{S}_{n}(2) \supset \cdots$ coincides with the lower central series of $\mathrm{IS}_{n}$.

For each $k \geq 1$, let $\mathcal{E}_{k}$ be a $\mathbf{Z}$-submodule of $H^{*} \otimes_{\mathbf{Z}} \mathcal{L}_{n}(k+1)$ consisting of all elements of type $x_{1}^{*} \otimes\left[B, x_{1}\right]$ where $B \in \mathcal{L}_{F}(k)$.

Lemma 4.1. For any $k \geq 1, \mathcal{E}_{k} \cong \mathcal{L}_{F}(k)$ as an abelian group.

Proof. Let $f_{k}: \mathcal{L}_{F}(k) \rightarrow \mathcal{E}_{k}$ be a homomorphism defined by $f_{k}(B):=x_{1}^{*} \otimes\left[B, x_{1}\right]$ for any $B \in \mathcal{L}_{F}(k)$. We construct the inverse of $f_{k}$ as follows. First, using a contraction, we define homomorphisms $\mu^{k}: H^{*} \otimes_{\mathbf{Z}} H^{\otimes(k+1)} \rightarrow H^{\otimes k}$ by

$$
x_{i}^{*} \otimes x_{j_{1}} \otimes \cdots \otimes x_{j_{k+1}} \mapsto-x_{i}^{*}\left(x_{j_{1}}\right) \cdot x_{j_{2}} \otimes \cdots \otimes x_{j_{k+1}}
$$

and

$$
\Phi^{k}:=\mu^{k} \circ\left(i d_{H^{*}} \otimes \iota_{n}^{k+1}\right): H^{*} \otimes_{\mathbf{Z}} \mathcal{L}_{n}(k+1) \rightarrow H^{\otimes k} .
$$

We denote the restriction of $\Phi^{k}$ to $\mathcal{E}_{k}$ by $g_{k}$. Then identifying $\mathcal{L}_{F}(k)$ with the image of a natural injective homomorphism $\iota_{k}: \mathcal{L}_{F}(k) \rightarrow H^{\otimes k}$, we obtain a homomorphism

$$
g_{k}: \mathcal{E}_{k} \rightarrow \mathcal{L}_{F}(k) .
$$

It is easily seen that $g_{k}$ is the inverse of $f_{k}$. Hence, we obtain the lemma. 
Next, let $\mathcal{T}_{k}$ be a $\mathbf{Z}$-submudule of $H^{*} \otimes \mathbf{z} \mathcal{L}_{n}(k+1)$ consisting of all elements of type $x_{1}^{*} \otimes A$ where $A \in \mathcal{L}_{F}(k+1)$. Clearly, we have $\mathcal{T}_{k} \cong \mathcal{L}_{F}(k+1)$ for any $k \geq 1$. Furthermore, from the elimination theorem of free Lie algebras, we see that the $\operatorname{sum} \mathcal{E}_{k}+\mathcal{T}_{k}$ in $H^{*} \otimes_{\mathbf{Z}} \mathcal{L}_{n}(k+1)$ as a $\mathbf{Z}$-module is a direct sum $\mathcal{E}_{k} \oplus \mathcal{T}_{k}$ for any $k \geq 1$. (See Proposition 10 in [3.) Then we show our main theorem.

Theorem 4.1. For each $k \geq 1$, we have $\mathcal{S}_{n}(k)=\mathcal{S}_{n}^{\prime}(k)$.

Proof. It suffices to show $\mathcal{S}_{n}(k) \subset \mathcal{S}_{n}^{\prime}(k)$ for each $k \geq 1$. For any $\sigma \in \mathcal{S}_{n}(k)$, by the split extension (4), there are some $v \in[V, V]$ and $w \in W$ such that $\sigma=v w$. Set $x_{1}^{v}:=x_{1} x$ and $x_{1}^{w}:=y^{-1} x_{1} y$ for $x, y \in F$. Then $x_{1}^{-1} x_{1}^{\sigma}=\left[x_{1}^{-1}, y^{-1}\right] x \in \Gamma_{n}(k+1)$.

First, we show $y \in \Gamma_{F}(k)$ and $x \in \Gamma_{F}(k+1)$. If $y \notin \Gamma_{F}(k)$, there is some $l \in\{1, \ldots, k-1\}$ such that $y \in \Gamma_{F}(l)$ and $y \notin \Gamma_{F}(l+1)$. Since both $\left[x_{1}^{-1}, y^{-1}\right]$ and $\left[x_{1}^{-1}, y^{-1}\right] x$ belong to $\Gamma_{n}(l+1)$, so does $x$. Hence we have

$$
\tau_{l}^{S}(\sigma)=x_{1}^{*} \otimes x_{1}^{-1} x_{1}^{\sigma}=x_{1}^{*} \otimes\left(\left[x_{1}^{-1}, y^{-1}\right] x\right)=x_{1}^{*} \otimes\left[x_{1}, y\right]+x_{1}^{*} \otimes x
$$

in $H^{*} \otimes \mathbf{z} \mathcal{L}_{n}(l+1)$. On the other hand, by $\left[x_{1}^{-1}, y^{-1}\right] x \in \Gamma_{n}(k+1) \subset \Gamma_{n}(l+2)$, we see $\tau_{l}^{S}(\sigma)=0$. Since $x_{1}^{*} \otimes\left[x_{1}, y\right] \in \mathcal{E}_{l}$ and $x_{1}^{*} \otimes x \in \mathcal{T}_{l}$ and since $\mathcal{E}_{l} \cap \mathcal{T}_{l}=0$ as mentioned above, we have $x_{1}^{*} \otimes\left[x_{1}, y\right]=x_{1}^{*} \otimes x=0$ in $H^{*} \otimes \mathbf{z} \mathcal{L}_{n}(l+1)$. Then, considering the isomorphism $g_{l}: \mathcal{E}_{l} \rightarrow \mathcal{L}_{F}(l)$ defined in (7), we obtain $y=0 \in \mathcal{L}_{F}(l)$. Hence $y \in \Gamma_{F}(l+1)$. This is a contradiction. Therefore we obtain $y \in \Gamma_{F}(k)$ and $x \in \Gamma_{F}(k+1)$. This shows that $\sigma \in \mathcal{S}_{n}^{\prime}(k)$ by the remark after Lemma 3.1 This completes the proof of Theorem 4.1

From Theorem 4.1 and Corollary 3.1, we have

Corollary 4.1. For each $k \geq 1, \operatorname{rank}_{\mathbf{Z}}\left(\operatorname{gr}^{k}\left(\mathcal{S}_{n}\right)\right)=r_{n-1}(k+1)+r_{n-1}(k)$.

\section{ACKNOWLEDGMENTS}

The author would like to express his sincere gratitude to the referee for helpful comments and correcting many typos and grammatical mistakes. This research is supported by a JSPS Research Fellowship for Young Scientists.

\section{REFERENCES}

[1] S. Andreadakis; On the automorphisms of free groups and free nilpotent groups, Proc. London Math. Soc. (3) 15 (1965), 239-268. MR0188307 (32:5746)

[2] Y. A. Bakhturin; Identities in Lie algebras, Nauka, Moscow 1985; English translation, Identical relations in Lie Algebras, VNU Science Press, Utrecht (1987). MR886063 (88f:17032)

[3] N. Bourbaki; Lie Groups and Lie Algebras, Chapters 1-3, Springer-Verlag (1989). MR979493 (89k:17001)

[4] F. Cohen and J. Pakianathan; On Automorphism Groups of Free Groups, and Their Nilpotent Quotients, preprint.

[5] F. Cohen and J. Pakianathan; On subgroups of the automorphism group of a free group and associated graded Lie algebras, preprint.

[6] B. Farb; Automorphisms of $F_{n}$ which act trivially on homology, in preparation.

[7] R. Hain; Infinitesimal presentations of the Torelli groups, Journal of the American Mathematical Society 10 (1997), 597-651. MR1431828 (97k:14024)

[8] D. Johnson; An abelian quotient of the mapping class group, Math. Ann. 249 (1980), 225-242. MR579103 (82a:57008)

[9] D. Johnson; The strucure of the Torelli group. III: The abelianization of $\mathcal{I}_{g}$, Topology 24 (1985), 127-144. MR793179 (87a:57016)

[10] N. Kawazumi; Cohomological aspects of Magnus expansions, preprint, arXiv:math.GT/ 0505497. 
[11] S. Krstić, J. McCool; The non-finite presentability in $I A\left(F_{3}\right)$ and $G L_{2}\left(\mathbf{Z}\left[t, t^{-1}\right]\right)$, Invent. Math. 129 (1997), 595-606. MR1465336 (98h:20053)

[12] W. Magnus; Über n-dimensinale Gittertransformationen, Acta Math. 64 (1935), 353-367. MR1555401

[13] W. Magnus, A. Karras, D. Solitar; Combinatorial group theory, Interscience Publ., New York (1966). MR2109550 (2005h:20052)

[14] J. McCool; Some remarks on IA automorpshims of free groups, Can. J. Math., Vol. XL, no. 5 (1998), 1144-1155. MR973513 (90d:20057)

[15] S. Morita; Abelian quotients of subgroups of the mapping class group of surfaces, Duke Mathematical Journal 70 (1993), 699-726. MR.1224104 (94d:57003)

[16] S. Morita; Structure of the mapping class groups of surfaces: a survey and a prospect, Geometry and Topology Monographs, Vol. 2, Geom. Topol. Publ., Coventry (1999), 349-406. MR.1734418 (2000j:57039)

[17] S. Morita; Cohomological structure of the mapping class group and beyond, Proc. of Symposia in Pure Math., 74, Amer. Math. Soc., Providence, RI (2006), 329-354. MR 2264550 (2007j:20079)

[18] J. Nielsen; Die Isomorphismen der allgemeinen unendlichen Gruppe mit zwei Erzeugenden, Math. Ann. 78 (1964), 385-397. MR1511907

[19] A. Pettet; The Johnson homomorphism and the second cohomology of $I A_{n}$, Algebraic and Geometric Topology 5 (2005) 725-740. MR 2153110 (2006j:20050)

[20] C. Reutenauer; Free Lie Algebras, London Mathematical Society Monographs, New Series, 7, Oxford University Press, New York (1993). MR 1231799 (94j:17002)

[21] T. Satoh; New obstructions for the surjectivity of the Johnson homomorphism of the automorphism group of a free group, Journal of the London Mathematical Society (2) 74 (2006), 341-360. MR2269583 (2007i:20060)

[22] E. Witt; Treue Darstellung Liescher Ringe, Journal für die Reine und Angewandte Mathematik, 177 (1937), 152-160.

Department of Mathematics, Graduate School of Science, Kyoto University, KitashirakawaOiWaKe-ChO, SAKYo-KU, KYOto City, 606-8502, Japan

E-mail address: takao@math.kyoto-u.ac.jp 